College of Technology; $\$ 275,000$ to the Western Region of Nigeria for the Institute of Administration at the University of Ife; and $\$ 125,000$ to the University of Dakar for research on African languages and improvement of facilities for English and French language instruction.

\title{
Centro de Estudios Africanos, Argentina
}

Following a series of seminars over three years at the National University of Buenos Aires and the Universidade de El Salvador, a Centre for African Studies has been established to provide courses, discussion meetings, and publications among teachers and students of the various institutions. The main concern of the Centre at the outset will be the political and constitutional aspects of African states, but it is expected that the range of interests will be broadened. 'The Centre would welcome opportunities to exchange information concerning work in African studies with other institutions. Communications should be addressed to the Centro de Estudios Africanos, Lavalle $1474,3^{\circ}, \mathrm{B}$, Buenos Aires, Argentina.

\section{Etude de la Flore Naturelle du Burundi}

MoYens employés : constitution d'un herbier conservé à l'Université officielle de Bujumbura, par écolte directe et par échange avec d'autres institutions de botanique africaine. Ce document scientifique permanent pourra être complété et amélioré au long de nombreuses années.

Intérêt de l'étude: la dorsale orientale de la crête Congo-Nil est occupée par des lambeaux de forêt en voie de disparition, dont les éléments sont jusqu'ici incomplètement connus. Il en est de même des prairies naturelles au-delà de $2.000 \mathrm{~m}$. Le but de cette étude phytosociologique est de mettre en évidence les étapes de la dégradation des forêts, les caractéristiques des diverses formations secondaires.

Étendue du sujet: le présent travail se doit de rassembler des documents sur les régions étudiées (Burundi, crête Congo-Nil) et sur toutes les régions limitrophes, afin de pouvoir établir d'une part des comparaisons, et d'autre part, les directions générales de l'évolution des formations forestières ou herbacées.

Trois études, par José Lewalle et F. M. Rodegem, sont en préparation: ' Plantes médicinales du Burundi': recension systématique et identification des plantes médicinales, á l'exclusion des plantes magiques; leur mode d'emploi, leurs indications thérapeutiques, leur récolte et leur usage. 'Les noms de plantes rundi': indications générales sur la nomenclature des plantes en langue rundi. Liste alphabétique des noms vernaculaires avec leur correspondance scientifique. 'Amulettes du Burundi': descriptions de certaines amulettes corporelles employées au Burundi dans un but bénéfique; leur variation, leur signification, leur distribution géographique.

(Communication de José Lewalle, Chargé d'enseignement, Faculté des Sciences, Laboratoire de Botanique, Université Officielle de Bujumbura)

\section{Patrimoine Culturel Rundi}

But de l'étude : établir un relevé aussi complet que possible de tous les éléments de la culture rundi en se basant sur les textes autochtones.

Matériel: transcription et traduction d'un choix de traditions orales ( $157^{2}$ pages polycopiées); textes inédits en voie de disparition, représentant les différents genres littéraires: fables, proverbes, contes et légendes, bestiaires, fabliaux, rondes populaires, épithalames, cantilènes et chansonnettes, le rire et l'humour, poésie, églogues et berceuses, chanson de geste, poésie épique. 